\title{
Observation of the fast NEO objects with prolonged exposure
}

\author{
O. Shulga, Y. Kozyryev and Y. Sibiryakova \\ Research Institute "Nikolaev Astronomical observatory" (RI NAO), Ukraine. \\ Email: tttt_nao@mail.ru
}

\begin{abstract}
An original way of application of drift-scan imaging - electronic tracing technique for observation of the fast moving asteroids is presented in this contribution.
\end{abstract}

Keywords. astrometry, solar system: general, techniques: miscellaneous

\section{Introduction}

There is a difficulty of observating the fast NEO objects with a speed $V>10$ " $\mathrm{sec}^{-1}$. That kind of objects cannot be observed with prolonged exposure as in classical method which is used for observing stars. Thus, the limiting magnitude of telescope cannot be reached. In order to increase the magnitude of observing objects a tracking mode is necessary. It is possible to have the following tracking modes:

- Digital - stacking of shifted images obtained with short exposure time;

- Mechanical - moving telescope around its two axes precisely;

- Electronic - tracing the electronic charge across the CCD chip.

The electronic tracking technique was designed in RI NAO. Observations are carried out when object is passing through the field of view of an unmovable telescope. Then, the exposure time depends on object's speed and a field size. This method can be used for observations of objects, ranging from stars to satellites in low Earth orbits.

\section{The electronic tracing technique}

The electronic tracing technique, designed in RI NAO, is based on Drift-Scan Imaging or Time Delayed Integration (TDI). The Drift-Scan Imaging technique is usually used to image long continuous strips of the sky. To make a scan it is necessary to read the lines of the CCD in perfect synchronization with the movement of the stars on the focal plane. It is also important to perfectly align the lines in the east-west direction. In RI NAO this technique has been used on Meridian Circle telescope since 1995. The field of application of the Drift-Scan Imaging technique can be extended considerably with possibility of rotation of the CCD camera to the certain angle. In that case it can be used for observation of any object which is uniformly moving through the field of view. For that purpose special rotation system was designed in RI NAO. The rotation system is a device in which a stepping motor and a angle encoder were equipped and which makes the rotation of CCD camera around optical axis of telescope.

\section{Test observations of the fast NEO object}

The electronic tracing technique was tested on very small telescope so only relative advantage can be showed. The test of observing asteroid $2007 \mathrm{dt} 103$ was realize with this method. On 2007-08-03 the asteroid speed is $-3 . ' 7 / \mathrm{min}$ in right accession, $-12 . " 4 / \mathrm{min}$ 


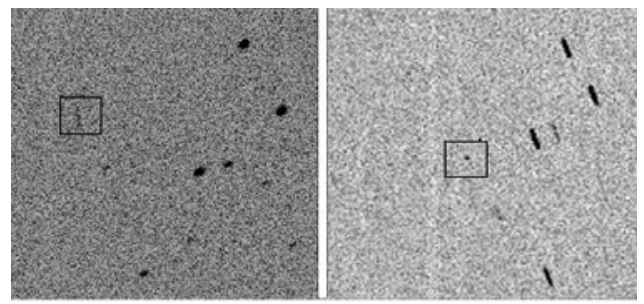

Figure 1. Images from different drift-scan observations. Left: Classical drift-scan imaging, right: Drift-scan with rotation system

in declination, and magnitude 14.1. The asteroid was observed on a unmovable telescope with the classical drift-scan imaging technique and with the rotation system (Figure 1). If exposure time is $170 \mathrm{sec}$, during the exposure time the asteroid passed $36 . " 6$ or 17 pixels on the focal plane.

\section{Drift-scan imaging with variable exposure time}

The other method to extend field of application of the Drift-Scan Imaging technique is to use variable exposure time. The exposure time of classical drift-scan method depends only on the speed of a target and field size which can't be changed (in full exposure time). But the way to use variable exposure time exists. During the specified exposure time CCD camera works in drift-scan mode (speed of reading out matches with the speed of a target and pixel scale). When the specified exposure time is over frame readout with maximum speed is started without CCD flushing. Variable exposure time is efficiently to use in the case if full exposure time is superfluous and good result can be obtained with much less exposure. The other advantage of variable exposure time is that several frames of the object can be obtained when it is passing through the CCD matrix.

\section{Additional reference stars frames}

Images obtained with object tracking mode (mechanical or electronic) contains round image of object but stretched images of stars which are difficult for data reduction. To avoid this problem the special additional frames with different mode are made before and after object frame. Telescope is unmovable so it is not a problem to connect the object coordinates and the reference stars coordinates obtained on different frames at different time. The easiest way to obtain reference stars frame on stare telescope is to use very short exposure time $(0.05-0.5 \mathrm{sec})$. When the fast NEO objects are observed with an unmovable telescope it is possible to use drift-scan imaging with variable short exposure time to obtain reference stars frame.

\section{Conclusion}

The electronic tracking technique can provide results comparable to that obtained by means of a precise tracking telescope for observing various types of objects, such as fast NEO, fast satellites in low Earth orbit and others. The majority of telescopes in Ukraine and Russia which are used for asteroid observations are old and cannot track such objects. The easiest and inexpensive way of modernization for tracking capability is to install the rotating system and CCD camera which supports the drift-scan mode. RI NAO in collaboration with Shanghai Astronomical Observatory carries out a joint project to use the electronic tracking technique. 